\title{
MULTI-OBJECTIVE DYNAMIC OPTIMIZATION OF AMPICILLIN BATCH CRYSTALLIZATION: SENSITIVITY ANALYSIS OF ATTAINABLE PERFORMANCE VS. PRODUCT QUALITY CONSTRAINTS
}

\author{
Antonios Dafnomilis ${ }^{\mathrm{a}}$, Samir Diab ${ }^{\mathrm{b}}$, Alistair D. Rodman ${ }^{\mathrm{b}}$, \\ Andreas G. Boudouvis ${ }^{\mathrm{a}}$, Dimitrios I. Gerogiorgis ${ }^{\mathrm{b} *}$ \\ ${ }^{\text {a }}$ School of Chemical Engineering, National Technical University of Athens, Athens 15780, Greece \\ ${ }^{\mathrm{b}}$ Institute for Materials and Processes (IMP), School of Engineering, University of Edinburgh, \\ The Kings Buildings, Edinburgh, EH9 3FB, Scotland, UK \\ *Corresponding Author: D.Gerogiorgis@ed.ac.uk
}

\section{SUPPORTING INFORMATION}

Figs. S1-S6 show optimal pH manipulation trajectories, supersaturation profiles and Mean Crystal Size (MCS) evolutions for different seed loading (Cases 1-3), the final ampicillin concentration constraint $\left([A m p]_{\text {target }}\right)$, the number of discretization elements $(N)$ and objective function component weights $(W)$. 


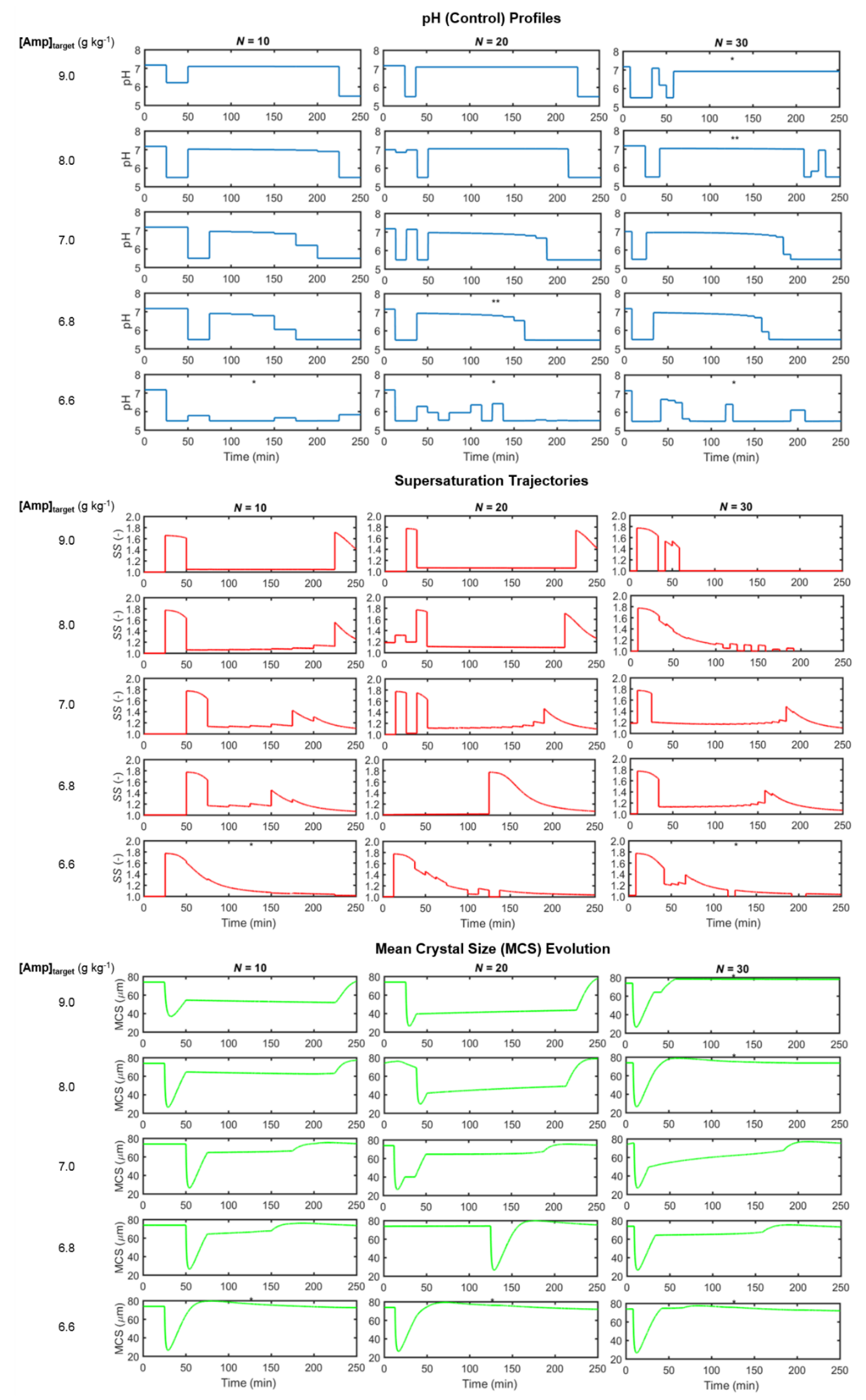

Figure S1: Optimization results for Case 1. Objective function weights: $W_{S T D}=1.0, W_{M C S}=0.5$; state variable collocation points: $K_{\mathrm{x}}=3$; initialization profile: $\mathrm{pH}(t)=7\left(*\right.$ : initialized at constant $\left.\mathrm{pH}(t)=6,{ }^{*}: K_{\mathrm{x}}=6\right)$. 


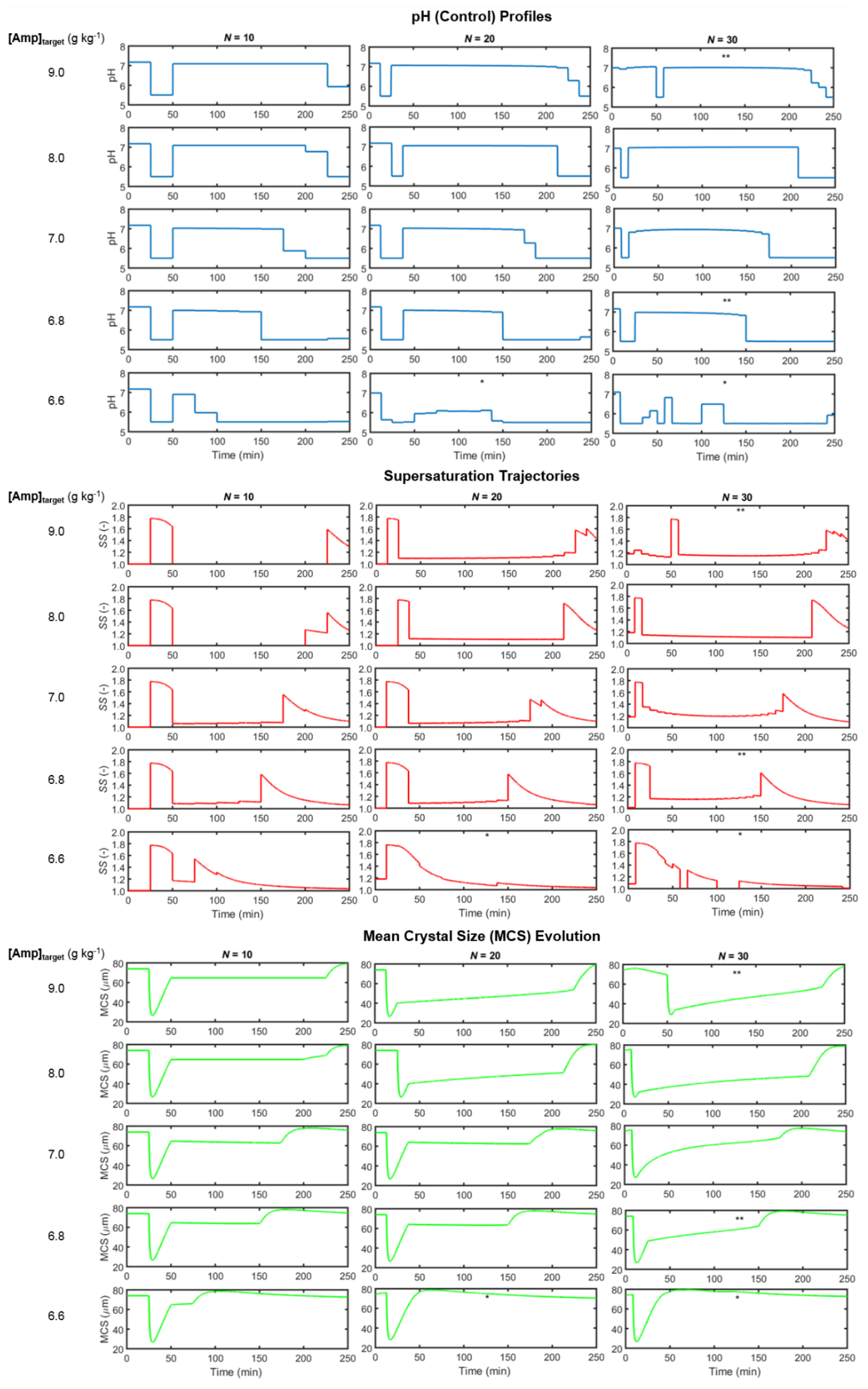

Figure S2: Optimization results for Case 1. Objective function weights: $W_{S T D}=1.0, W_{M C S}=1.5$; state variable collocation points: $K_{\mathrm{x}}=3$; initialization profile: $\mathrm{pH}(t)=7\left(*\right.$ : initialized at constant $\left.\mathrm{pH}(t)=6,{ }^{*}: K_{\mathrm{x}}=6\right)$. 

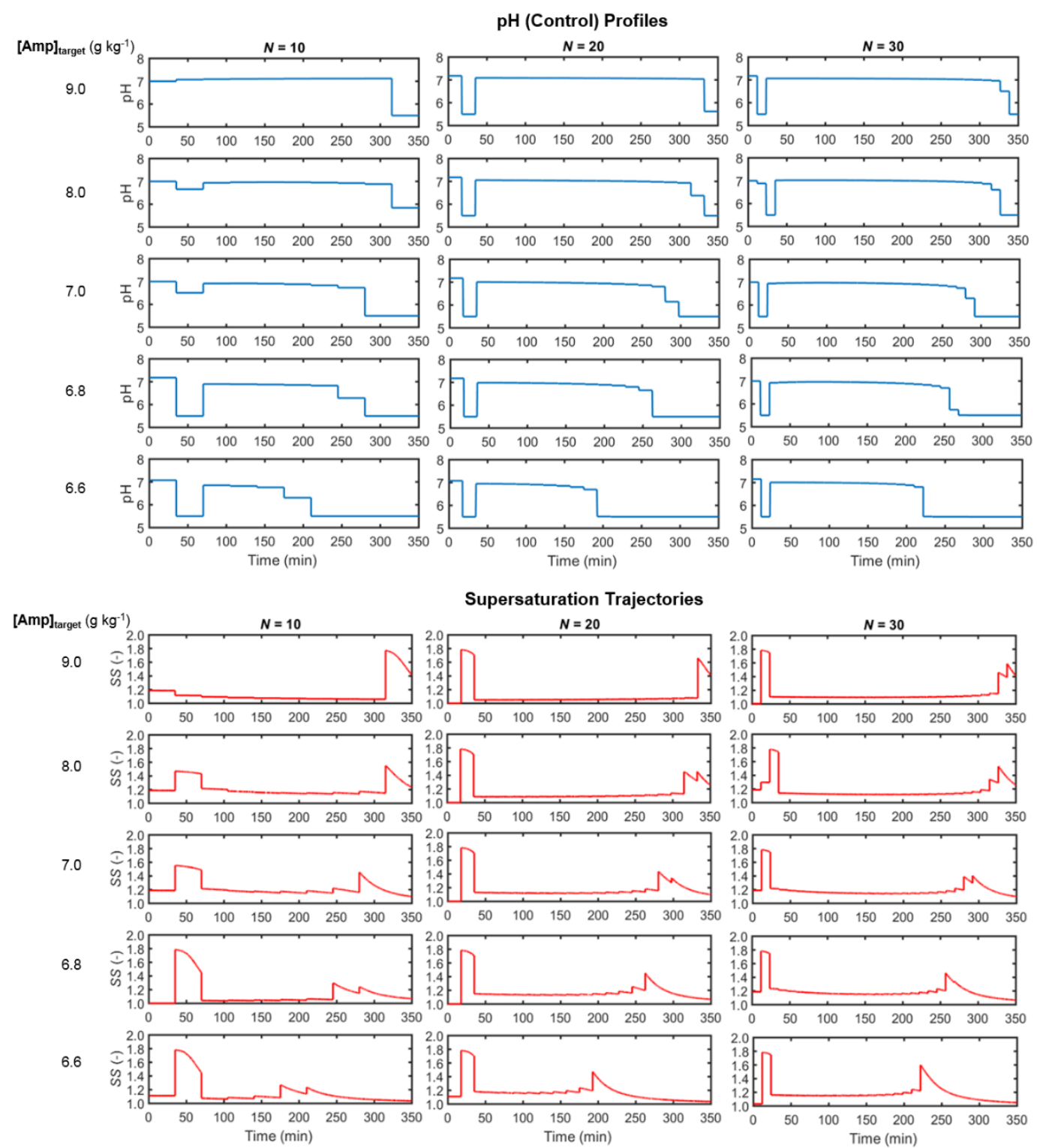

Supersaturation Trajectories
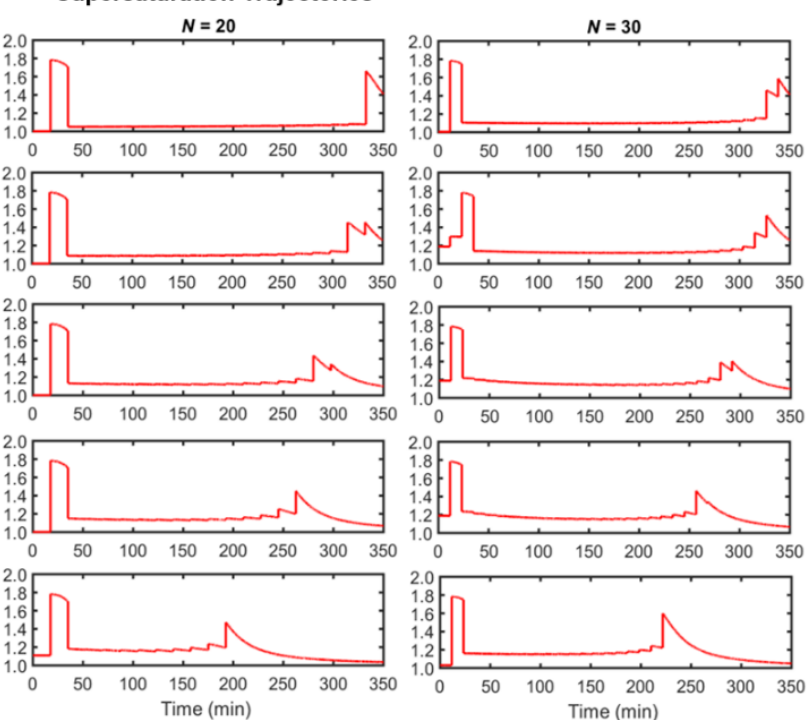

\section{Mean Crystal Size (MCS) Evolution}
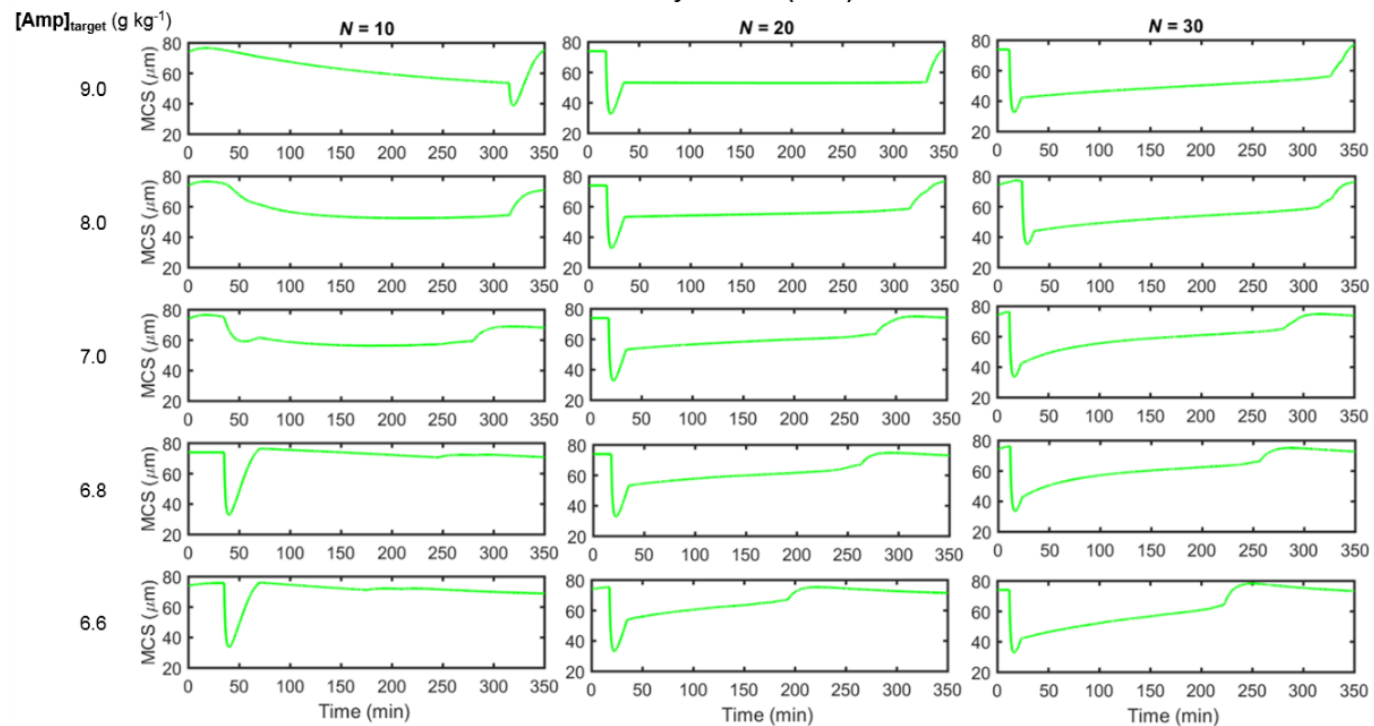

Figure S3: Optimization results for Case 2. Objective function weights: $W_{S T D}=1.0, W_{M C S}=0.5$; state variable collocation points: $K_{\mathrm{x}}=3$; initialization profile: $\mathrm{pH}(t)=7$. 

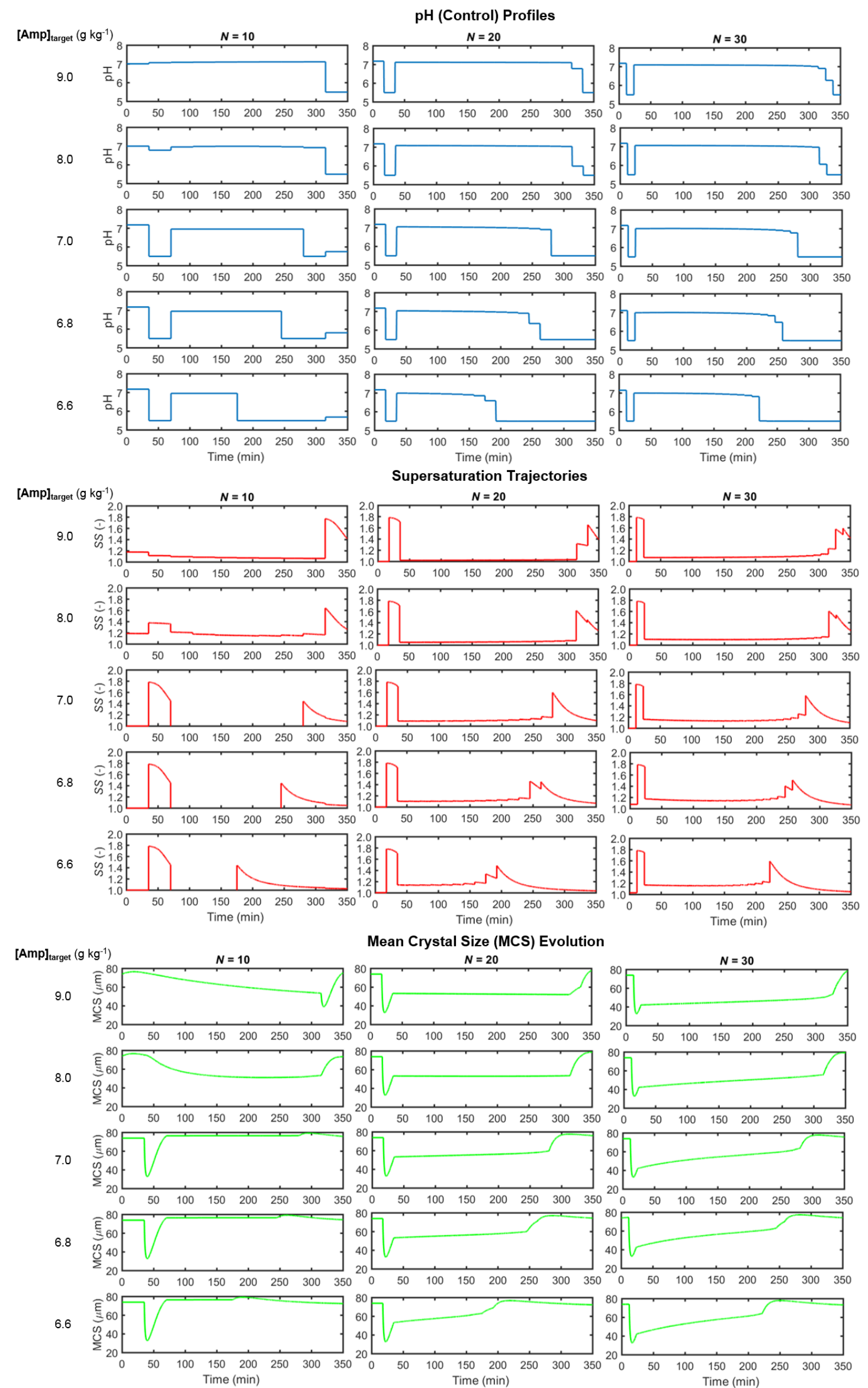

Mean Crystal Size (MCS) Evolution
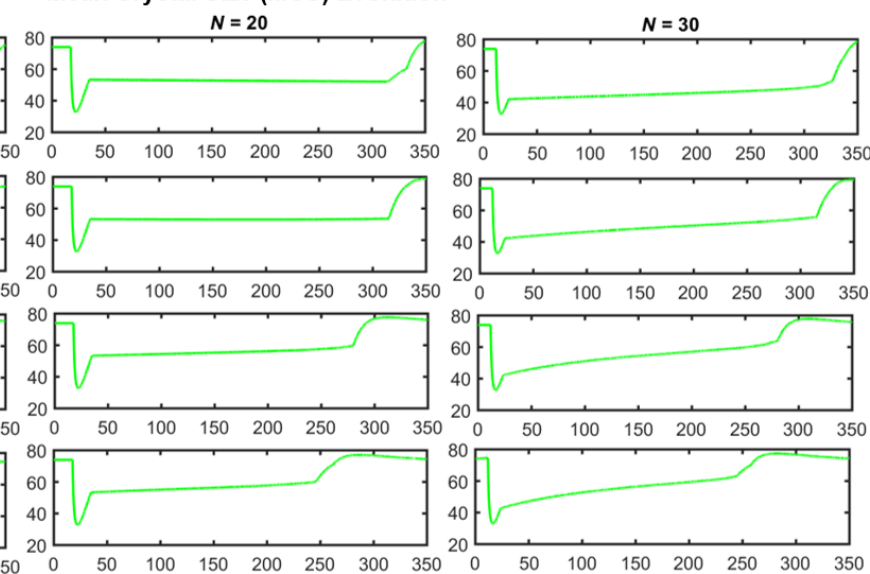

Figure S4: Optimization results for Case 2. Objective function weights: $W_{S T D}=1.0, W_{M C S}=1.5$; state variable collocation points: $K_{\mathrm{x}}=3$; initialization profile: $\mathrm{pH}(t)=7$. 


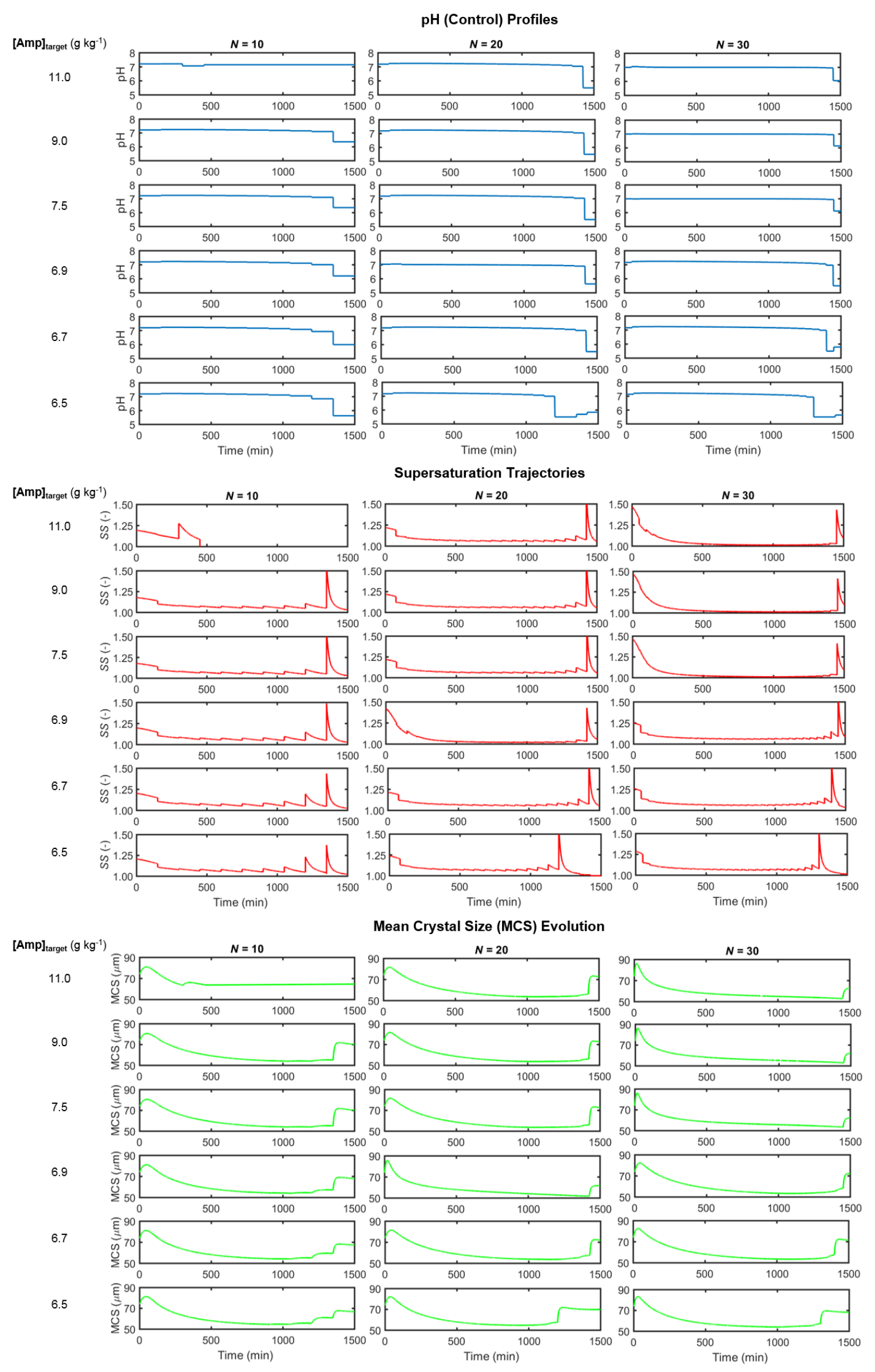

Figure S5: Optimization results for Case 3. Objective function weights: $W_{S T D}=1.0, W_{M C S}=0.5$; state variable collocation points: $K_{\mathrm{x}}=3$; initialization profile: $\mathrm{pH}(t)=7$. 
$\mathrm{pH}$ (Control) Profiles
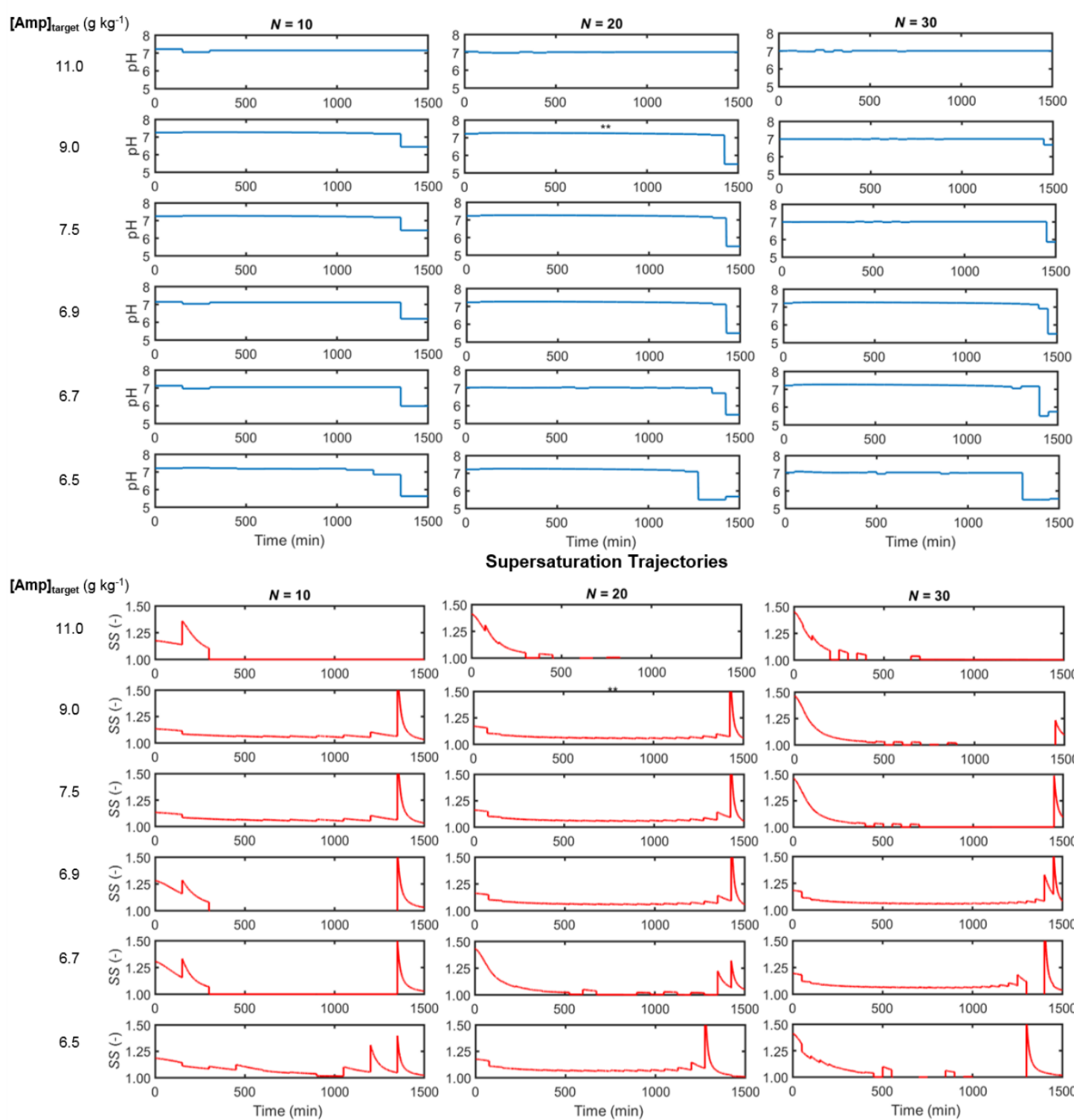

Supersaturation Trajectories
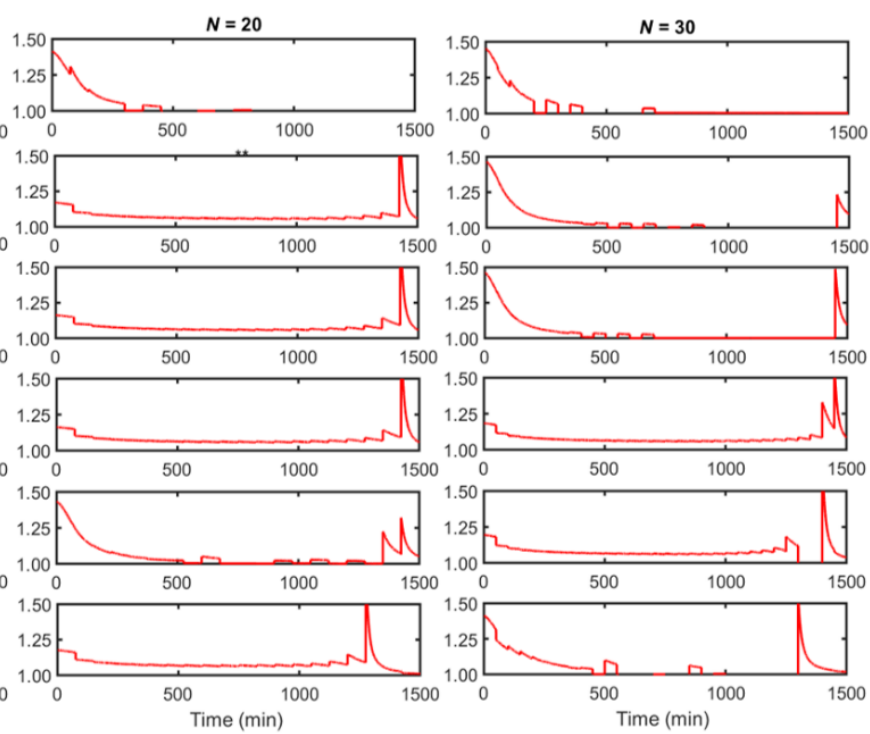

Mean Crystal Size (MCS) Evolution
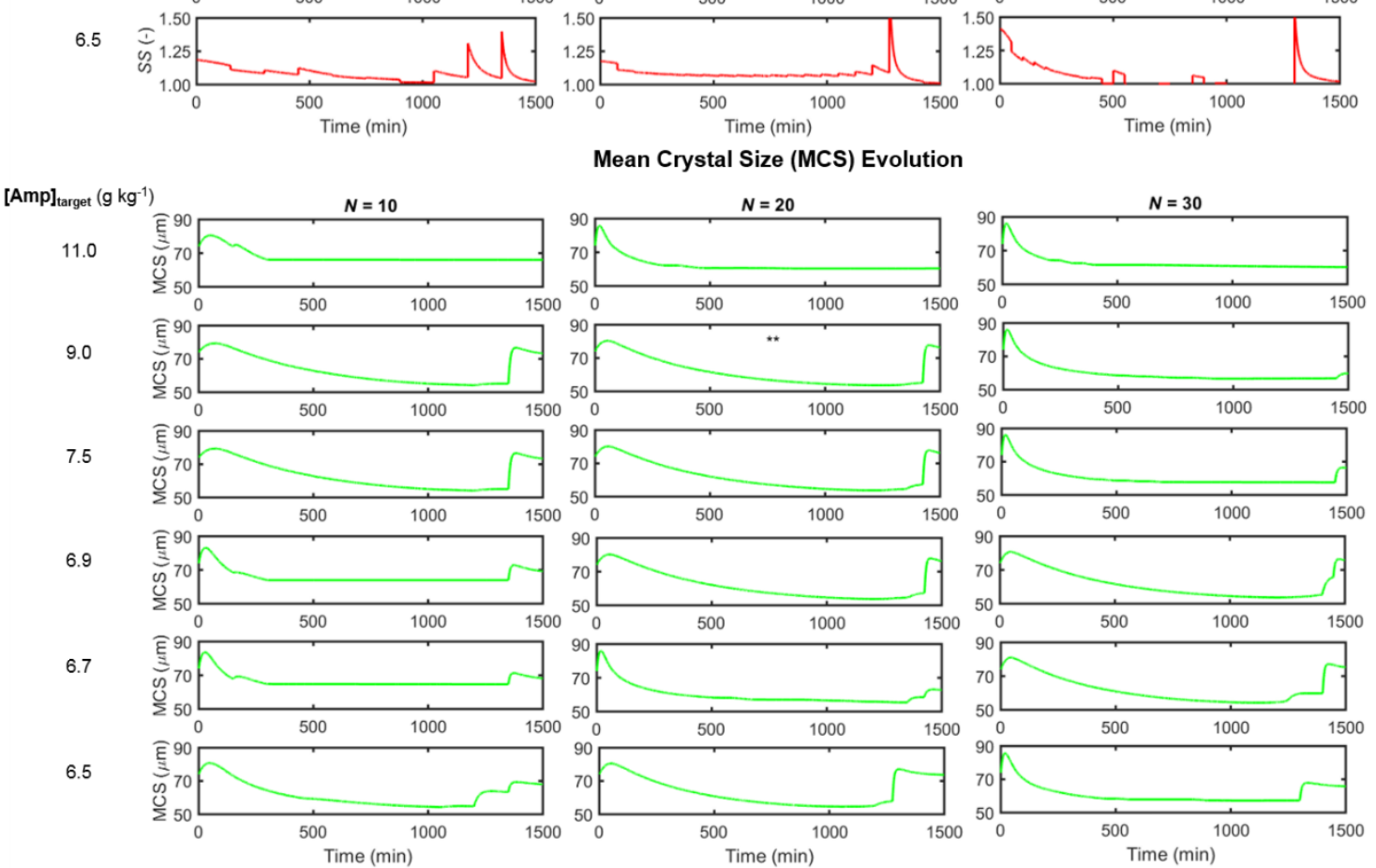

Figure S6: Optimization results for Case 3. Objective function weights: $W_{S T D}=1.0, W_{M C S}=1.5$; state variable collocation points: $K_{\mathrm{x}}=3$; initialization profile: $\mathrm{pH}(t)=7$. 